7. Child Lang. 29 (2002), $25 \mathrm{I}-274$. (C) 2002 Cambridge University Press

DOI : Io.IoI7/So305000902005032 Printed in the United Kingdom

\title{
Restructuring of similarity neighbourhoods in the developing mental lexicon*
}

\author{
HOLLY L. STORKEL \\ Indiana University \\ (now at the University of Kansas) \\ (Received 3 October 2000. Revised 6 Fune 200 I)
}

\begin{abstract}
A BSTRACT
Previous evidence suggests that the structure of similarity neighbourhoods in the developing mental lexicon may differ from that of the fully developed lexicon. The similarity relationships used to organize words into neighbourhoods was investigated in 20 pre-school children (age $3 ; 7$ to 5 ; I I) using a two alternative forced-choice classification task. Children classified the similarity of test words relative to a standard word to determine neighbourhood membership. The similarity relationship between the test and standard words varied orthogonally in terms of type of similarity and position of overlap. Standard words were drawn from neighbourhoods differing in density. Results showed that dense neighbourhoods were organized by phoneme similarity in the onset + nucleus or rhyme positions of overlap. In contrast, sparse neighbourhoods appeared to be organized by phoneme similarity in the onset + nucleus, but manner similarity in the rhyme. These results are integrated with previous findings from infants and adults to propose a developmental course of change in the mental lexicon.
\end{abstract}

\section{INTRODUCTION}

Studies of the mental representation of concepts have generally shown that categories change over the course of development (see Smith \& Samuelson, I 997 for review). In particular, children initially appear to categorize concepts holistically with membership based on overall similarity; whereas later in development, categorization appears analytic with membership based

[*] This research was supported by grants from the National Institutes of Health to Indiana University, Bloomington (DC ooo I 2, DC or 694, DC 0478I). We appreciate the insightful comments and assistance provided by Judith Gierut throughout this project. Toby Calandra, Annette Champion, and Michele Morrisette aided in the recruitment of participants. We would also like to thank two anonymous reviewers who provided helpful comments on a previous version of this manuscript. Address for correspondence: Holly L. Storkel, PhD, Department of Speech-Language-Hearing, 300 I Dole Human Development Center, University of Kansas, Lawrence, KS 66045, USA. e-mail: hstorkel $@$ ku.edu 
on similarity on particular dimensions (Treiman \& Baron, I98 г ; Treiman \& Breaux, I982; Walley, Smith \& Jusczyk, I986; Gattuso, Smith \& Treiman, I 99 I; Gerken, Murphy \& Aslin, I 995; Gierut, I 996; but see also Ward \& Vela, I 986). This developmental sequence has been observed across both non-linguistic and linguistic categories. For example, in non-linguistic domains, when asked to select visually similar stimuli varying in colour and size or overall similarity, categorization based on similarity in the colour and size dimensions increased from kindergarten to adulthood (Gattuso et al., I99I). Turning to linguistic domains, Gierut (I996) observed that some preschool children classified fricative sounds (e.g. /f $\theta \mathrm{s} /$ ) based on the feature [continuant], but that children with a fuller productive repertoire of fricatives classified these same sounds based on a combination of the features [continuant] and [distributed]. Changes in sound categories and changes in the productive use of these same sounds were thus associated. Taken together, it appears that acquisition of new knowledge seems to promote changes in the mental representation of that knowledge. The purpose of this investigation is to examine the mental representation of the phonological properties of words. Lexical acquisition certainly involves adding new words to the existing mental lexicon, but it is unclear whether the mental representation of words changes with development. We begin by reviewing the current evidence regarding the mental representation of words, beginning with the fully developed adult lexicon and then turning to the developing lexicon.

\section{REPRESENTATION OF WORDS IN THE MENTAL LEXICON}

It has been suggested that the phonological properties of words in the fully developed lexicon are organized into lexical similarity neighbourhoods commonly defined as words differing from a target word by a one phoneme substitution, deletion, or addition in any word position (Luce \& Pisoni, I 998). That is, the neighbours of a consonant-vowel-consonant (CVC) word would include all the words that share two to three phonemes with the target word. For example, the neighbours of 'top' would include 'toss,' 'tap,' 'cop,' and 'stop.' Words that are similar to only a few other words are said to reside in sparse neighbourhoods; whereas words that are phonologically similar to many other words are considered to reside in dense neighbourhoods. There are several important assumptions regarding the mental representation of words inherent in this definition of neighbourhood. First, it is assumed that the type of similarity used to organize words into neighbourhoods is phoneme similarity. Second, neighbourhoods purportedly consist of words representing three positions of overlap: onset + nucleus $\left(\mathrm{CV}_{-}\right)$, onset + coda (C_C), and nucleus + coda or rhyme (_VC). Onset + nucleus overlap refers to words that share the initial consonant(s) and the vowel (e.g. ' $r a t$ ' and ' $r a p$ '). Onset + coda overlap is the condition where words have the same initial and 
final consonants (e.g. 'rat' and 'root'). Rhyme overlap occurs when words share the same vowel and final consonant(s) (e.g. ' rat' and 'hat'). Lastly, in the fully developed lexicon, it is assumed that lexical structure is similar across neighbourhoods. The finding that the number of neighbours influences word recognition in adults has supported the psychological reality of this neighbourhood structure. In particular, words from dense neighbourhoods are recognized more slowly and less accurately than words from sparse neighbourhoods (see Luce \& Pisoni, r 998 for review).

Turning to the developing mental lexicon, computational studies suggest that the mental representation of words may not parallel that of the fully developed lexicon. These studies involved first identifying a data source representative of the lexicon of particular age groups of children. This is followed by calculation of neighbourhood density that assumes a particular organizational structure with comparisons then across different age groups (Charles-Luce \& Luce, I 990, I 995; Logan, I 992; Dollaghan, I994). Such computational studies based on both expressive and receptive databases have demonstrated that words in the lexicons of one- to seven-year-old children are less dense than those of an adult, and that density increases over time (Charles-Luce \& Luce, I 990, I 995 ; Dollaghan, I 994). The words in a young child's lexicon may be more distinct with fewer neighbours than the same words in the fully developed lexicon. As a result, children may be able to rely on more holistic representations to uniquely differentiate each word from every other, and these representations may become more detailed as words are acquired and density increases (Charles-Luce \& Luce, I990, I995; Metsala \& Walley, ı998; Garlock, Walley \& Metsala, 200 i; but see Dollaghan, I994). Thus, as new words are acquired, the mental representation of known words may change to resemble the lexical structure of an adult. In considering this hypothesis, there are at least three possible ways in which the structure of the developing lexicon might differ from that of the fully developed lexicon: ( I) type of similarity for neighbourhood membership; (2) position of overlap for neighbourhood membership; (3) consistency of structure across neighbourhoods. These follow directly from the basic assumptions of lexical organization reviewed above.

\section{Type of similarity}

Evidence of the type of similarity used to structure similarity neighbourhoods in the developing lexicon comes from two sources: computational studies of production and behavioural studies of perception. Given that words are more distinct in the child than the adult lexicon, it is possible that a less detailed representation, such as one based on manner or place similarity, may sufficiently differentiate each word from every other in the child lexicon. Considering first the computational evidence based on production, Logan 
( I992) examined lexical representations in one- to five-year-old children by comparing density calculations based on three types of similarity: phoneme, manner, and place. To illustrate, if the developing lexicon were organized by phoneme similarity, then the representation of ' rat' would be $/ \mathrm{r} æ \mathrm{t} /$, and neighbours would presumably include words sharing any two of the three phonemes. Alternatively, if a child's lexicon is organized by manner similarity, then the representation of 'rat' is proposed to be /liquid æ stop/, and neighbours would include other words sharing two of the three specified manners. Finally, if a child's lexicon is organized by place similarity, then the representation of ' rat' is proposed to be / palatal æalveolar/, and neighbours would include words sharing two of these three places of articulation. Logan's ( I 992) comparison of density in lexical acquisition based on each type of similarity showed that organization by manner did not appreciably alter density from organization by phoneme. Therefore, it is possible that a less segmentally detailed organization by manner may be sufficient to uniquely differentiate each word in the developing lexicon from every other. In contrast, organization by place increased the density of children's neighbourhoods when compared to organization by phoneme. Thus, if place similarity is used to structure a child's lexicon, this may cause greater confusion among similar sounding words.

Evidence from behavioural studies of perception complements Logan's (r992) computational findings. Jusczyk, Goodman \& Baumann (I999) showed that infants preferred to listen to lists of words sharing manner, rather than lists of words sharing place of articulation. This finding again suggests that manner may be a more salient feature than place in the developing organization of the lexicon. Likewise, results from classification tasks have shown that preschool children experience difficulties categorizing nonwords that share phonemes, tending instead to group nonwords based on overall similarity (Treiman \& Baron, I98 I ; Treiman \& Breaux, I 982; Walley et al., I 986; but see Gerken et al., I 995). Given this, neighbourhoods in the developing mental lexicon may not be structured around phoneme similarity (Metsala \& Walley, I 998). It is important to note that previous behavioural work has typically employed nonword stimuli (but see Cole, r98r). Nonwords and novel words, by definition, are not yet members of the lexicon, and presumably have no lexical representation prior to their first introduction. Consequently, it is unclear to what extent the previous findings reflect the representation of real words in the developing mental lexicon.

\section{Position of overlap}

There are other differences between children and adults related to sensitivity to position of overlap (e.g. Elliott, Hammer \& Evan, I 987; Walley, I 988). In production, babble and first words tend to favour CV over CVC structures 
(see Stoel-Gammon \& Dunn, I985, for review). This observation suggests that children may initially represent the onset+nucleus rather than the rhyme position of overlap. Likewise, perceptual studies indicate that infants recognize similarity in words overlapping in the onset + nucleus position, but not in the rhyme position (Jusczyk et al., i 999; Swingley, Pinto \& Fernald, I 999). In addition, Walley et al. (i 986) showed that five- and six-year-old children were able to identify phonological similarity in word initial syllables but not in word final syllables; whereas, seven- and eight-year-old children identified similarity in either word position. In contrast, classification studies provide evidence that children may first identify similarity in the rhyme position, rather than the onset (e.g. Treiman \& Zukowski, I99I). Taken together, past findings suggest that the developing mental lexicon may be organized into neighbourhoods based on overlap in only one position, but there is controversy regarding which position of overlap, onset + nucleus or rhyme, may be used to structure neighbourhoods early in development.

\section{Consistency of structure}

Finally, changes in the mental representation of words are likely to occur gradually, paralleling other forms of development (Ferguson \& Farwell, I975; Stoel-Gammon \& Cooper, I984). This may result in asymmetries across dense versus sparse neighbourhoods. Neighbourhood density may promote change in the lexical representations of those particular words that are more likely to be confused with other words, namely words from dense neighbourhoods (Metsala \& Walley, I998). For example, assuming that neighbourhoods are initially organized on the basis of manner similarity, the lexical representation for the dense word 'sit' would be /fricative I stop/. As other words are learned, it is likely that this representation of 'sit' would produce confusion with other phonologically similar words that would have the same representational characteristics, such as 'fit' and 'sick' (Dollaghan, I994). Conversely, a sparse word 'these', having the representation /fricative i fricative/, would tend to have less potential for confusion because only a few (likely unknown) words, such as 'thief' and 'seize', would have the same representation (Dollaghan, I994). In support of this hypothesis, adults and children show similar word recognition performance for dense words, but show differing word recognition performance for sparse words (Metsala, I997; Garlock et al., 2001). It is possible that these asymmetries in word recognition may be attributable to asymmetries in lexical structure, with dense words in the developing lexicon being structurally similar to those in the fully developed lexicon, but sparse words being structurally different from those in the fully developed lexicon.

The purpose of the current study was to examine the representation of words in preschool children using a behavioural classification task. Building 
upon the prior research, we tested three hypotheses. First, similarity neighbourhoods in children may not be based on phoneme similarity, but rather, more coarsely coded similarity such as shared manner or place. Second, similarity neighbourhoods in children may include words overlapping in only one position, excluding words overlapping in the alternate position. Finally, structural organization may be asymmetric, varying across sparse and dense neighbourhoods. To test these hypotheses, we investigated the type of similarity (identical vs. phoneme vs. manner vs. place) and position of overlap (onset + nucleus vs. rhyme) used to organize neighbourhoods differing in density (sparse vs. dense).

\section{METHODS}

Participants

Twenty typically developing preliterate preschool children $(M=4 ; 8$; range 3;7-5; I I) participated. $^{1}$ All children were monolingual native English speakers, and had normal development by parent report on a questionnaire. Additionally, hearing, articulation, and receptive vocabulary were screened prior to participation in the experimental task to ensure that all children performed within normal limits for their age. Participants passed an audiometric screening (ASHA, I985) and scored at the 32 nd percentile or above on the Goldman-Fristoe Test of Articulation ( $M=68$ th percentile; Goldman \& Fristoe, I 986) and the Peabody Picture Vocabulary Test-Revised ( $M=72$ nd percentile; Dunn \& Dunn, I98I). In addition, children were required to accurately produce the experimental stimuli. The lexical representation of misarticulated words is controversial, with some proposing lexical representations similar to the adult target and others proposing representations similar to the child's surface production (Smith, I973; Macken, I980; Dinnsen \& Elbert, I984). For this reason, we limited the stimuli to only correctly articulated words. Each stimulus item was elicited one time in a story re-telling task. Child productions were phonetically transcribed, and judged correct if all target phonemes were produced as intended in the ambient language.

\section{Stimuli}

CVC real words were selected as stimuli to examine the following lexical contrasts: neighbourhood density (sparse vs. dense), type of similarity (identical vs. phoneme vs. manner vs. place), and position of overlap

[I] Given that a wide age range was used, the data were examined for age effects by dividing the participants into an older and younger group using a median split of chronological age. No differences were identified between these two groups so the data are reported as an aggregate $(F<2 \cdot 0$ for the main effect and all interactions of age with other variables). 
(onset + nucleus vs. rhyme). Three dense and three sparse words were selected as STANDARDS for comparison to potential neighbours. For each standard, eight TEST WORDS were identified as potential neighbours to examine four types of similarity (identical, phonemic, manner, or place) across two positions of overlap (onset + nucleus, or rhyme). It was anticipated that responses to the identical similarity condition would be near $100 \%$, and thus this condition was included as a reference point for determining attention to the task.

CVC stimuli were selected from a computerized dictionary consisting of 20,000 words (Nusbaum, Pisoni \& Davis, I 984). Density was calculated by counting the number of words in the dictionary differing from a given word by a one-phoneme substitution. A median split was used to divide sparse from dense words. To guard against misarticulation of the stimuli, CVC words composed of sounds acquired after age 4; o (Smit, Hand, Freilinger, Bernthal \& Bird, I990) were eliminated from the pool of candidates leaving approximately I 70 sparse and 237 dense words as potential standards. Words subjectively judged to be unknown by young children were also eliminated, leaving 54 sparse and 65 dense standards. For these remaining standards, potential test words were then generated to determine whether the full array of type of similarity $\times$ position of overlap conditions could be filled. Potential standards were eliminated from consideration if a test word could not be identified for each type of similarity $\times$ position of overlap condition in a mutually exclusive way. Final standards and test words were selected from the remaining pool so that manner and place characteristics were replicated across standards from sparse and dense neighbourhoods and across test

TA B LE I . Phonetic transcription of standards varying in neighbourhood density (sparse vs. dense) and associated tests words examining position of overlap (onset +nucleus vs. rhyme) and type of similarity (identical vs. phoneme vs. manner vs. place)

\begin{tabular}{|c|c|c|c|c|c|c|}
\hline & \multicolumn{3}{|c|}{ Sparse standards } & \multicolumn{3}{|c|}{ Dense standards } \\
\hline & $/ \mathrm{t} \wedge \mathrm{g} /$ & /daim/ & /bum/ & /tæp/ & $/ \operatorname{tin} /$ & /dip/ \\
\hline \multicolumn{7}{|l|}{ Onset + nucleus overlap } \\
\hline Identical match & $/ \mathrm{t} \wedge \mathrm{g} /$ & / daIm/ & /bum/ & /tæp/ & $/ \mathrm{t} \operatorname{In} /$ & / $\mathrm{dip} /$ \\
\hline Phoneme similarity & $\mathrm{t} \wedge \mathrm{f}$ & $\overline{\mathrm{daId}}$ & $\overline{\text { but }} /^{-}$ & $\overline{\operatorname{tæn}} /$ & $\overline{\mathrm{tIk}} /$ & $/ \overline{\mathrm{dI} \breve{s}} /$ \\
\hline Manner similarity & $\overline{\mathrm{b} \wedge} \mathrm{s} /$ & / $\overline{\text { baIt }} /$ & /gus / & /pæn/ & /pIg/ & / $\overline{\mathrm{kI}} /$ \\
\hline Place similarity & $\underline{\mathrm{s} \wedge \mathrm{n} /}$ & nait/ & fud $/$ & /sæy/ & / $\underline{\text { sIk }} /$ & /sIy/ \\
\hline \multicolumn{7}{|l|}{ Rhyme overlap } \\
\hline Identical match & $/ \underline{\mathrm{t}} \wedge \mathrm{g} /$ & / & / bum/ & /tæp/ & / $\underline{\operatorname{tIn}} /$ & /dip/ \\
\hline Phoneme similarity & $/ \overrightarrow{\mathrm{h} \wedge \mathrm{g} /}$ & $/ \overline{\mathrm{maIm}}$ & $\overline{\text { rum }} /$ & $/ \mathrm{mæp} /$ & $/ \overline{\mathrm{wIn}} /$ & $/$ šp/ \\
\hline Manner similarity & $/ \mathrm{m} \wedge \mathrm{d} /$ & $/ \mathrm{maIn} /$ & /sun/ & $/$ fæt $/$ & $/ \mathrm{h} \overline{\mathrm{Im}} /$ & $/ \mathrm{ft} /$ \\
\hline Place similarity & $/ \mathrm{j} \wedge \overline{\mathrm{n}}^{\prime}$ & /waIp/ & $/ \overline{\sup } /$ & $/ \mathrm{hæm} /$ & $/ \mathrm{h} \underline{\underline{\mathrm{t}} /}$ & $/ \overline{\mathrm{hIm}} /$ \\
\hline
\end{tabular}


words differing in position of overlap (onset + nucleus vs. rhyme). Stimuli are shown in Table $\mathrm{r} .^{2}$

Previous studies have shown that density is often correlated with other lexical or phonological factors. Given that correlated factors may influence responding, the density, phonotactic probability, perceptual confusability, familiarity and word frequency of the stimuli were explored to determine if these factors varied systematically across the independent variables. First, the density of the test words was examined. This factor was not controlled because the neighbourhood structure of the standard word was the primary

T А В LE 2. Density, segment frequency, and biphone frequency for test words in each type of similarity and position of overlap condition

\begin{tabular}{|c|c|c|c|c|c|c|}
\hline & \multicolumn{3}{|c|}{ Sparse standards } & \multicolumn{3}{|c|}{ Dense standards } \\
\hline & Density & $\begin{array}{l}\text { Segment } \\
\text { frequency }\end{array}$ & $\begin{array}{l}\text { Biphone } \\
\text { frequency }\end{array}$ & Density & $\begin{array}{l}\text { Segment } \\
\text { frequency }\end{array}$ & $\begin{array}{l}\text { Biphone } \\
\text { frequency }\end{array}$ \\
\hline \multicolumn{7}{|c|}{ Type of similarity } \\
\hline \multicolumn{7}{|c|}{ Identical match } \\
\hline$M$ & I9 & 0.076 & 0.00 I 8 & 27 & 0.142 & 0.0068 \\
\hline S.D. & I & O.OI I & 0.0008 & 3 & 0.028 & 0.0047 \\
\hline \multicolumn{7}{|c|}{ Phoneme similarity } \\
\hline$M$ & 20 & 0.076 & 0.0018 & 23 & 0.142 & 0.0068 \\
\hline S.D. & 7 & O.OI I & 0.0008 & 7 & 0.028 & 0.0047 \\
\hline \multicolumn{7}{|c|}{ Manner similarity } \\
\hline$M$ & 20 & 0.092 & 0.0024 & 23 & O. I 64 & 0.0057 \\
\hline S.D. & 6 & 0.029 & 0.0016 & 7 & 0.018 & 0.0022 \\
\hline \multicolumn{7}{|c|}{ Place similarity } \\
\hline$M$ & I 7 & 0.075 & 0.0023 & 25 & O. I 69 & 0.0069 \\
\hline S.D. & 8 & 0.034 & 0.0019 & 5 & 0.042 & 0.0022 \\
\hline \multicolumn{7}{|c|}{ Position of overlap } \\
\hline \multicolumn{7}{|c|}{ Onset + nucleus } \\
\hline$M$ & I 6 & 0.082 & 0.0022 & 22 & O. I 69 & 0.0068 \\
\hline S.D. & 7 & 0.026 & ०.०० 8 & 7 & 0.035 & 0.0043 \\
\hline \multicolumn{7}{|l|}{ Rhyme } \\
\hline$M$ & I 4 & 0.080 & 0.00 I I & 25 & 0.148 & 0.0062 \\
\hline S.D. & 7 & 0.027 & 0.0012 & 5 & 0.022 & ०.00 I 5 \\
\hline
\end{tabular}

focus. Table 2 shows the density of the test words by neighbourhood density, type of similarity, and position of overlap. The test words for standards from dense neighbourhoods tended to be higher in density than those from sparse neighbourhoods, $t(34)=-2.06 \mathrm{I}, p<0.05$. Within sparse and dense stan-

[2] Note that 'him' was used in the manner similarity condition for the dense standard 'tin' and in the place similarity condition for the dense standard 'dip.' The responses to each individual series of standards and test words were visually examined and judged to be similar across series. Thus, the use of 'him' in two different conditions does not appear to have influenced responding. 
dards, the density of the test words was similar across types of similarity (all comparisons $t(\mathrm{I} O)<\mathrm{I}$ ) and positions of overlap (all comparisons $t(\mathrm{I} 6)<\mathrm{I}$ ).

Second, the phonotactic probability of the standards and test words was computed because past work has shown that these two variables are positively correlated in English (Vitevitch, Luce, Pisoni \& Auer, I 999). PHONOTACTIC PROBABILITY refers to the likelihood of occurrence of sound sequences in a language and is based on two measures: positional segment frequency and biphone frequency (Vitevitch \& Luce, I999). Positional SEGMENT FREQUENCY is the likelihood of occurrence of a given sound in a given syllable position. BIPHONE FREQUENCY is the likelihood of occurrence of a given sound preceding or following another sound. These frequencies were computed using the previously described on-line dictionary and were weighted for word frequency. Density was significantly correlated with positional segment frequency, $r=0.606, p<0.00 \mathrm{I}$, and biphone frequency, $r=0.404, p<0.0 \mathrm{I}$. Table 2 shows the phonotactic probability of the segments in the target position of overlap (onset + nucleus, rhyme), excluding from the calculation the non-overlapping position. Test words for dense standards had higher segment frequencies and higher biphone frequencies than those for sparse standards, $t(34)=-8.177, p<0.00 \mathrm{I}$ and $t$ (34) $=-5.28 \mathrm{I}, p<0.00 \mathrm{I}$ respectively. Within sparse and dense standards, the phonotactic probability of the test words was similar across types of similarity (all comparisons $t(\mathrm{I} \mathrm{O})<\mathrm{I} \cdot 5)$ and positions of overlap (all comparisons $t(\mathrm{I} 6)<\mathrm{I} \cdot 5)$.

Third, the perceptual confusability of test words and standards was computed using position specific phoneme confusion matrices obtained for adults (Luce, I986). For each test word, the probability of identifying the phoneme from the standard word when presented with the phoneme from the test word at a + I 5 signal-to-noise ratio was computed. For example, the probability of confusing the /b/ in the standard word 'boom' with the /g/ in the test word 'goose' was calculated. Considering first the phonemes in the non-overlapping positions, the probability of confusing the phoneme from the standard with that of the test word was extremely low (probability of ०.००-०.०I). This further supports the mutual exclusivity of the position of overlap conditions by showing that standard and test words were only similar in the intended position of overlap. Turning to the phonemes in the overlapping positions, the confusability of each overlapping element was computed and then multiplied to yield one probability (e.g. p('boom '|'goose') $=\mathrm{p}(\mathrm{b} \mid \mathrm{g}) \times \mathrm{p}(\mathrm{u} \mid \mathrm{u})$. Confusability was similar across sparse and dense neighbourhoods $(t(34)<\mathrm{I})$. Confusability did vary by type of similarity. The standards and test words that shared phonemes had higher confusability (probability of $0.39^{-0.8 \mathrm{I}}$ ) than those that shared either manner or place (probability of ०.० I-०.I5; all comparisons $t$ ( $\left.\mathrm{I}_{0}\right)>9.5, p<0.00 \mathrm{I}$ ). This is to be expected because the probability of identifying /bu/ in 'boom' when 
given /bu/ in 'boot' represents the likelihood of correctly identifying the target phonemes. Importantly, the test words that shared either manner or place had low confusability with the standard. Thus, on both linguistic and perceptual grounds, only test words sharing phonemes with the standards would be expected to be neighbours of the standards in the fully developed lexicon. Confusability was similar across positions of overlap (all comparisons $t($ I 6$)<\mathrm{I})$.

Fourth, given that classification of nonwords may not reflect the organization of lexical representations (Vitevitch \& Luce, I 999), the familiarity of the standard and test words was examined using expressive and receptive databases representative of two- to three-year-old children (Brown, I973; Rescorla, I989; Reznick \& Goldsmith, I989). Eighty-three percent of the stimuli were found in these databases, suggesting that young children were likely to know the majority of the standard and test words. Furthermore, the stimuli that could not be found in a database were not concentrated in a given neighbourhood density $\times$ type of similarity $\times$ position of overlap condition. The familiarity of the stimuli was further verified for each participant in the story re-telling task (see procedure section below).

Lastly, word frequency was determined by using log frequency based on Kolson's ( I 960/ I 962) database of children's spoken language. Seven of the words were not found in the child frequency database. These words were equally distributed across conditions. Word frequency did not differ significantly across the levels of neighbourhood density (means of $2 \cdot 88-3.06$; $t(29)<\mathrm{I}$ ), type of similarity (means of $2 \cdot 49-3 \cdot 28$; all comparisons $t$ ( Iо) $<\mathrm{I}$. 8 ), or position of overlap (means of $2 \cdot 7 \mathrm{I}-3 \cdot 22$; all comparisons $t(\mathrm{I} 4)<\mathrm{I}$ ).

To summarize, the test words for dense standards tended to be higher in density and phonotactic probability than the test words for sparse standards, but confusability, familiarity, and word frequency were similar across the dense and sparse stimuli. Thus, density was confounded with phonotactic probability; however, previous studies have shown that density effects tend to emerge for real words, and phonotactic probability effects tend to emerge for nonwords (Vitevitch \& Luce, I 999). Given that the stimuli were familiar real words, it is hypothesized that neighbourhood density will be the relevant variable influencing performance. Across the levels of type of similarity, only perceptual confusability showed systematic variation, verifying that correct identification of shared phonemes was likely and confusion of different phonemes was unlikely. Relative to position of overlap, there were no significant differences between the onset + nucleus and the rhyme positions of overlap on any other factor.

Stimuli were digitized, edited and recorded on a master tape. To ensure adequate clarity of the recorded stimuli, two listeners, blind to the stimulus items, phonetically transcribed the words. Both listeners transcribed $100 \%$ of the stimuli as intended. 
Task

Children participated in a two alternative forced-choice classification game involving a small garbage can and a character bank (Gerken et al., I995; Gierut, I 996). The master tape was played at a comfortable listening level by desktop speakers. The standard word for each comparison was introduced as the character bank's favourite word. The child was instructed to place a chip in the bank every time the standard word or a word like the standard word was heard (see appendix). This response was scored as 'neighbour of the standard'. If the word was not like the standard word, then the child was instructed to place the chip in the garbage can. This response was scored as 'not a neighbour of the standard'. No feedback as to the accuracy of responding was provided to avoid biasing the child to respond in a particular way.

\section{Procedures}

Children participated in three sessions. The first session consisted of standardized testing to determine eligibility, as previously described. During this session, children also were familiarized with the experimental stimuli in a story, and then spontaneous use of the stimuli was evaluated by having the child re-tell the story. The purpose of this manipulation was to assess articulation of each stimulus, verify that the stimuli were familiar, and provide exposure to the referent of any unfamiliar stimuli. Children spontaneously produced $70 \%$ (range $50 \%-80 \%$ ) of the words, verifying that the majority of the stimuli were familiar to the participants.

The two alternative forced-choice classification task was administered over an additional two sessions. Each experimental session began with a pretraining period to familiarize the child with the task. There were three graded phases in this pretraining process that varied the phonological relationship between standard and test words and the conceptual association between the character bank and the standard word. In phase I, children classified test items that IDENTICALLY MATCHED the standard word (e.g. 'fish' - 'fish') or bore no phonological resemblance to the standard word (e.g. 'moon' - 'fish'). The association between the standard word and the character bank was concrete because the standard word named the character bank (i.e. 'fish' paired with a fish bank). In phase 2, children classified test items that were PHONEMICALLY SIMILAR to the standard word (e.g. 'woo' - 'boo') or bore no phonological resemblance to the standard word (e.g. 'oink' - 'boo'). Phonemically similar items shared the same vowel. Here, the association between the standard word and the character bank was more abstract with the standard word being thematically related to the character bank (i.e. 'boo' paired with a ghost). In phase 3 , children classified test items that were FEATURALly SIMILAR to the standard (e.g. 'night' - 'knife') or shared no 
TABLE 3. Results of the repeated measures ANOVA with Huynh-Feldt adjustment for sphericity (Huynh $\wp^{\circ}$ Feldt, I976) and measures of effect size

\begin{tabular}{|c|c|c|c|c|c|c|}
\hline \multirow[b]{2}{*}{ Variable } & \multirow[b]{2}{*}{$\mathrm{df}^{a}$} & \multicolumn{3}{|c|}{ ANOVA results } & \multicolumn{2}{|c|}{ Effect size } \\
\hline & & & $F$ & $P$ & $f^{b}$ & $P V^{c}$ \\
\hline \multicolumn{7}{|l|}{ Main effects } \\
\hline Type of similarity & 3 & 57 & $107 \cdot 234$ & $\leqslant 0.0 I^{*}$ & $3 \cdot 93$ & 0.80 \\
\hline Position of overlap & I & I 9 & 2.639 & $>0.05$ & 0.14 & 0.12 \\
\hline Neighbourhood density & I & I9 & $58 \cdot 120$ & $\leqslant 0.0 I^{*}$ & 3.06 & 0.75 \\
\hline \multicolumn{7}{|l|}{ 2-Way interactions } \\
\hline Type of similarity $\times$ position of overlap & 3 & 57 & $9 \cdot 066$ & $\leqslant 0.0 I^{*}$ & 0.40 & $0 \cdot 28$ \\
\hline Type of similarity $\times$ neighbourhood density & 3 & 57 & $3 \cdot 454$ & $\leqslant 0.05^{*}$ & 0.19 & $0 \cdot 16$ \\
\hline Position of overlap $\times$ neighbourhood density & I & 19 & $4 \cdot 295$ & $\leqslant 0.05^{*}$ & 0.23 & O. I 8 \\
\hline 3-Way interaction & & & & & & \\
\hline $\begin{array}{l}\text { Type of similarity } \times \text { position of overlap } \\
\times \text { neighbourhood density }\end{array}$ & 3 & 57 & $4 \cdot 600$ & $\leqslant 0.0 I^{*}$ & 0.20 & 0.17 \\
\hline
\end{tabular}

a Degrees of freedom for the variable of interest and the error term. Note that children had to pass the pre-training criterion for each stimulus set (onset + nucleus vs. rhyme) administered on different days. Two children passed the pre-training on one day, but failed on another day. Specifically, one child completed only the rhyme stimulus set and another completed only the onset + nucleus stimulus set. For the ANOVA analysis, the missing data were replaced with ' $O$ ' as suggested by Weiss (I 99 I).

$b$ The effect size $f^{2}$ is the standard deviation of standardized means. Values $\leqslant 0.02$ are considered small effects; values $=0 . I_{5}$ are considered medium effects; values $\geqslant 35$ are considered large effects (Cohen, I988).

${ }^{c}$ A measure of effect size detailing the proportion of the variance in responding accounted for by the variable. Values $\leqslant 0^{\circ} \circ \mathrm{I}$ are considered small effects; values $=0 \cdot$ I 0 are medium effects; values $\geqslant 0.25$ are large effects (Cohen, I988).

phonemes with the standard word (e.g. 'wash' - 'knife'). Featurally similar items shared the same vowel and contained consonants sharing manner and/or place features with the standard word, directly paralleling the stimuli to be used in the experimental task. In this phase, the standard word was conceptually unrelated to the character bank (e.g. ' knife' paired with a bear). To advance to the experimental task, children were required to demonstrate differential responding to the similar and dissimilar items by classifying $50 \%$ or more of the similar items as 'neighbours of the standard' and $50 \%$ or more of the dissimilar items as 'not a neighbour of the standard'.

Following successful completion of pretraining, children advanced to the experimental task. Test stimuli focusing solely on the onset + nucleus position of overlap $(n=24)$ and those focusing solely on the rhyme position of overlap $(n=24)$ were presented on different test days. The order of administration of these two stimulus sets was counterbalanced across participants. For each position of overlap (onset + nucleus vs. rhyme), each of the six standards and the associated test words were presented in a block of trials with sparse and dense standards alternating in $\mathrm{I}$ of 2 presentation 


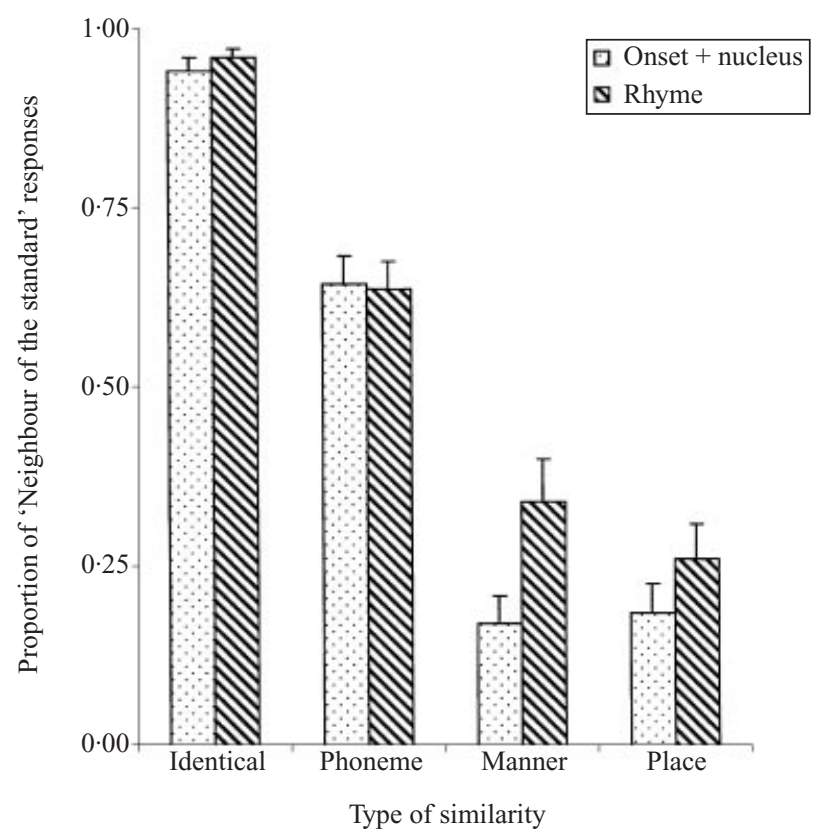

Fig. I. Proportion of 'neighbour of the standard' responses for standard words from dense neighbourhoods. Test words varied in type of similarity, identical versus phoneme versus manner versus place, and position of overlap, onset + nucleus (flecked bar) versus rhyme (striped bar).

orders counterbalanced across participants (Gerken et al., I995; Gierut, I996). In a block of trials, a given standard was introduced at the start of the block, and then test words examining the four types of similarity (identical vs. phoneme vs. manner vs. place) were presented for classification. Each test item was presented four times in randomized order for a total of 16 trials. The standard was repeated after 8 trials to refresh the child's memory. The child was given 5 seconds between test items to make a response.

RESULTS

The proportion of 'neighbour of the standard' responses out of the total number of responses was computed for each condition and submitted to a (4) type of similarity (identical vs. phoneme vs. manner vs. place) $\times(2)$ position of overlap (onset + nucleus vs. rhyme) $\times(2)$ neighbourhood density (sparse vs. dense) repeated measures analysis of variance with Huynh-Feldt adjustment for sphericity (Huynh \& Feldt, I976). To provide a more detailed analysis of neighbourhood structure, significant effects were explored by comparing the obtained values for each relevant condition to chance performance using a binomial test with Bonferroni adjustment for multiple 
comparisons. The purpose of this analysis was to determine if the obtained values were significantly above chance $(0.50)$, indicating membership in the neighbourhood, or significantly below chance, indicating exclusion from the neighbourhood.

Results of the repeated measures ANOVA are shown in Table 3. With the exception of the main effect of position of overlap, all main effects and twoway interactions were significant $(p \leqslant 0.05)$. Moreover, the three-way interaction of type of similarity (identical, phoneme, manner, place) $\times$ position of overlap (onset + nucleus, rhyme) $\times$ neighbourhood density (sparse, dense) was significant, $F(3,57)=4.60 ; p \leqslant 0.0 \mathrm{I}$. This three-way interaction was explored by comparing the obtained values for each type of similarity $\times$ position of overlap $\times$ neighbourhood density condition to chance performance. Figure I displays the proportion of 'neighbour of the standard' responses for standard words from dense neighbourhoods. Responses to the onset + nucleus position of overlap are shown by the flecked bar, and those to the rhyme position of overlap are shown by the striped bar. Considering the onset + nucleus position of overlap, words identically matching or sharing phonemes with the standard were classified significantly above chance as neighbours of the dense standard $(p \leqslant 0.00 \mathrm{I})$. In contrast, words sharing manner or place class in the onset + nucleus position were classified significantly below chance $(p \leqslant 0.00 \mathrm{I})$, indicating that these words were not neighbours of the dense standards. The rhyme position of overlap showed a similar pattern of neighbourhood membership. Words identically matching or sharing phonemes with the standard were classified significantly above chance as neighbours of the dense standard $(p \leqslant 0.00 \mathrm{I})$; whereas words sharing manner or place class were classified significantly below chance as being neighbours of the dense standard $(p \leqslant 0.00 \mathrm{I})$. These findings from development directly parallel previous claims about the fully developed lexicon; namely, dense neighbourhoods are largely organized by phoneme similarity in either the onset + nucleus or the rhyme position of overlap.

Figure 2 displays the proportion of 'neighbour of the standard' responses for standard words from sparse neighbourhoods. Here, a different pattern was observed for the onset + nucleus position as compared to the rhyme position of overlap. In the onset + nucleus position of overlap, words identically matching or sharing phonemes with the sparse standards were classified significantly above chance as neighbours $(p \leqslant 0.00 \mathrm{I})$, and words sharing manner or place were classified significantly below chance as neighbours $(p \leqslant 0.00 \mathrm{I})$. Findings from the onset + nucleus neighbours of sparse words thus paralleled those from dense words. In the rhyme position of overlap, however, words identically matching, sharing phonemes, or sharing manner class with the standard were classified significantly above chance as neighbours of the sparse standards $(p \leqslant 0.00 \mathrm{I})$. Only words sharing place with the standard were classified significantly below chance as 


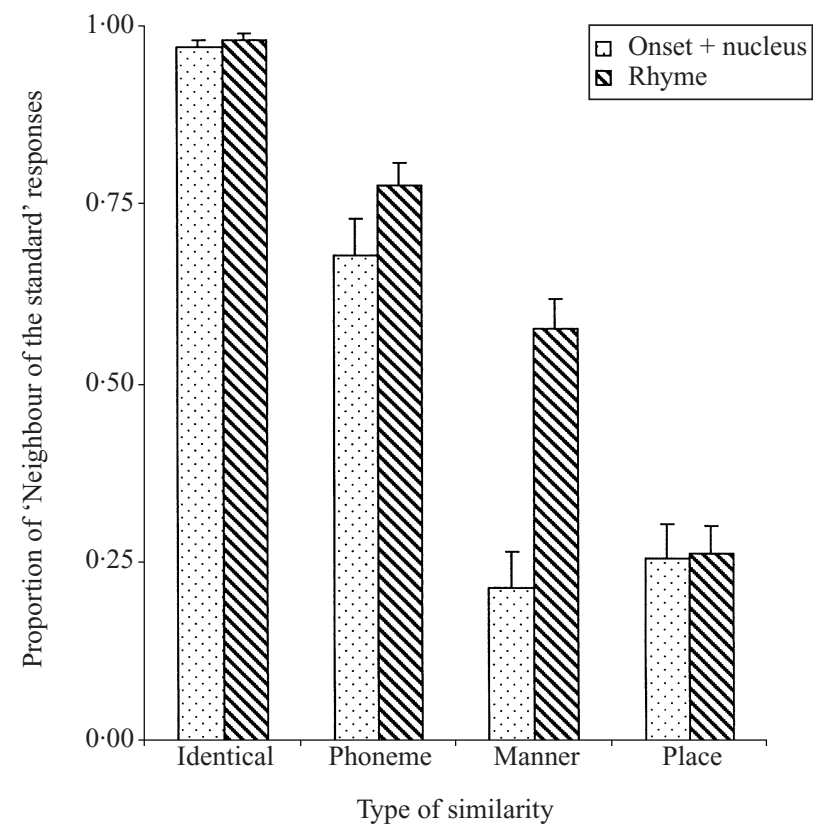

Fig. 2. Proportion of 'neighbour of the standard' responses for standard words from sparse neighbourhoods. Test words varied in type of similarity, identical versus phoneme versus manner versus place, and position of overlap, onset + nucleus (flecked bar) versus rhyme (striped bar).

neighbours of the sparse standards $(p \leqslant 0.001){ }^{3}$ The structure of sparse neighbourhoods appeared to include phoneme or manner similarity in the rhyme position, which differed from the structure of rhyme neighbours of dense words.

DISCUSSION

This study investigated the structure of the developing mental lexicon by examining the type of similarity (phoneme vs. manner vs. place) and position of overlap (onset + nucleus vs. rhyme) used to form neighbourhoods differing in density (sparse vs. dense). The underlying hypotheses were that the developing mental lexicon may be organized along coarse similarity dimensions, may define neighbourhood membership based on overlap in only one

[3] Each type of similarity by position of overlap condition also were compared across sparse and dense neighbourhoods. These pairwise comparisons complemented the analysis comparing obtained performance to chance. In particular, the only significant differences were found in the rhyme position of overlap where test words sharing manner similarity were selected more frequently as neighbours of sparse standards than as neighbours of dense standards $(F(\mathrm{I}, \mathrm{I} 9)=44.058 ; p<0.00 \mathrm{I})$. 
position, and may be structurally asymmetric across sparse and dense neighbourhoods. Support was found for each of these hypotheses. The results showed that membership in dense neighbourhoods seemed to be based on phoneme similarity in either the onset+nucleus or the rhyme position. In contrast, membership in sparse neighbourhoods seemed to be based on phoneme similarity in the onset + nucleus, but manner similarity in the rhyme. That is, sparse neighbourhoods seemed to have less detailed segmental representations of the rhyme than dense neighbourhoods.

These findings are consistent with the LEXICAL RESTRUCTURING MODEL (LRM) proposed by Metsala \& Walley (1998). This model claims that children's lexical representations are initially holistic but gradually become more segmental as words are acquired. In fact, our results along with past results, indicate that the type of similarity used to structure the lexicon changes from manner class similarity to phoneme similarity (Jusczyk et al., I999). Moreover, the LRM proposes that words in dense neighbourhoods are more prone to restructuring than words in sparse neighbourhoods because of greater potential for confusion among words in dense neighbourhoods. Thus, the LRM accounts for the obtained asymmetry in the structure of dense versus sparse neighbourhoods. Our results extend the LRM by providing further evidence of the role of position of overlap in restructuring. In particular, the onset + nucleus appeared more vulnerable to restructuring than the rhyme. One possible explanation for this result relates to the temporal nature of spoken language. In particular, sounds at the beginning of a word are heard first and may serve as cues in processing subsequent sounds in the word (Gupta \& Dell, 1997). For this reason, confusability in the onset + nucleus may have a greater impact on language processing than confusability in the rhyme; therefore, there may be greater pressure to develop a segmentally detailed representation of the onset + nucleus.

While there is evidence to support the LRM, what remains less clear is how restructuring occurs. There are two possible mechanisms that may account for developmental changes in representations (see Carey, 1985). The strong restructuring account assumes that neighbourhoods are reorganized as greater detail is incorporated into lexical representations. The weak restructuring account assumes that neighbourhood membership is static across development, but that attention to particular similarity relationships among neighbours shifts across development (Nittrouer, 1996; Smith \& Samuelson, 1997). Each of these accounts will be considered in turn.

\section{Strong restructuring account}

Considering first the strong restructuring account, Figure 3 provides a schematic of the proposed changes in neighbourhood membership across infants (left panel), preschool children (middle panel), and adults (right panel). We consider 'boom', which resides in a sparse neighbourhood, and 


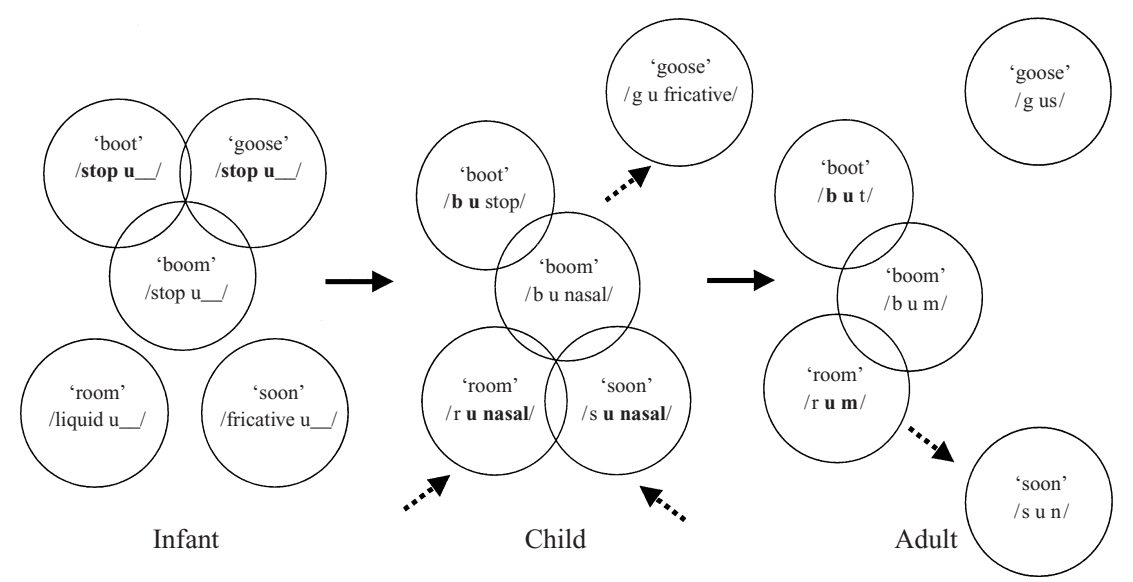

Fig. 3. Changes predicted by the strong restructuring account for the sparse neighbourhood 'boom' in the infant (left panel), preschool child (middle panel), and adult (right panel) lexicons. Similarity relationships are noted in bold.

its potential onset + nucleus neighbours, 'boot' (shared phoneme) and 'goose' (shared manner), as well as potential rhyme neighbours, 'room' (shared phoneme) and 'soon' (shared manner). In infancy, lexical representations are presumably less detailed, with neighbourhood membership thought to be organized by manner similarity in the onset + nucleus position only (Jusczyk et al., I 999; Swingley et al., I 999). As in the left-most panel of Figure 3, 'boot' and 'goose' would, therefore, be neighbours of 'boom' because these share the onset + nucleus /stop u /. In contrast, 'room' and 'soon' would reside in different neighbourhoods because these overlap with 'boom' only in the rhyme, and presumably the rhyme is not lexically represented at this stage. We hypothesize that this asymmetry between the onset + nucleus and the rhyme is attributable to the sequential nature of spoken language (Gupta \& Dell, i 997).

During childhood, we suggest from our results that the type of similarity and position of overlap for neighbourhood membership changes because the lexicon increases in size. We hypothesize that phonemic coding emerges in the onset + nucleus position to avoid confusion among neighbours overlapping in this position. This change in type of similarity presumably entails the movement of some former neighbours out of the neighbourhood. In the middle panel of Figure 3, notice that the representation of the onset + nucleus for 'boom', 'boot', and 'goose' has changed from /stop u / to / b u/, /b u/, and /g u/ respectively. Because the representation of 'goose' is no longer similar to the representation of 'boom' in terms of onset + nucleus overlap, 'goose' moves out of the neighbourhood. In contrast, 'boot' remains in the neighbourhood because it shares the onset + nucleus /b u/ with 'boom.' 
During this same period, new words are also moving into the neighbourhood because the rhyme position of overlap becomes important for neighbourhood membership. We assume that there is pressure for full specification of the word to support accurate production and word recognition and decrease the confusability among words. The representation of the rhyme for 'boom,' 'room,' and 'soon' has changed from / u _ / to / u nasal/, and accordingly 'room' and 'soon' move into the neighbourhood for 'boom.'

In adulthood, as the lexicon continues to increase in size, the type of similarity used to organize neighbours overlapping in the rhyme position again changes. Neighbourhood membership is now thought to be based on phoneme similarity in either position of overlap to avoid confusion among similar words (e.g. Luce \& Pisoni, I 998). As in earlier stages, the emergence of phonemic coding is accompanied by exodus from the neighbourhood. In the right-most panel of Figure 3, the representation of the rhyme for 'boom,' 'room,' and 'soon' has changed from / $\mathrm{u}$ nasal/ to / $\mathrm{u} \mathrm{m} / \mathrm{,} / \mathrm{u} \mathrm{m} /$, and / u n/ respectively. 'Soon' no longer shares relevant properties with 'boom' because the type of similarity used to structure neighbourhoods has changed. As a result, 'soon' moves out of the neighbourhood. By adulthood, the mental lexicon is presumably characterized by detailed phonemic representations for words in sparse neighbourhoods. It is important to note that the assumption of homogeneous lexical structure across neighbourhoods differing in density has not been explicitly tested in adults. Past studies have focused on the effects of density on spoken language processing, rather than the effects of density on the structure of lexical representations. It is possible that sparse neighbourhoods may continue to have less detailed representations into adulthood. Future studies of lexical structure in adults across neighbourhoods differing in density may shed further light on this issue.

Thus far, we have traced a possible developmental restructuring of a sparse neighbourhood. Restructuring of dense neighbourhoods will predictably follow a similar path, but is likely to be accelerated because of the greater potential for confusion between phonologically similar words. For preschoolers, we observed that membership in dense neighbourhoods was already based on phoneme similarity in the onset + nucleus or rhyme position, the proposed structure for the fully developed lexicon. Presumably, dense neighbourhoods, like sparse neighbourhoods, would go through a period where membership is based on manner similarity in the rhyme position. This period may have been overlooked in this study because of the limited age range sampled. Continued investigations of younger children, two- and three-year-olds, may help to better delineate this developmental course of lexical restructuring in dense neighbourhoods. Extending this further, dense neighbourhoods may even be structured by phoneme similarity in the onset + nucleus or rhyme position as early as infancy. As in the adult literature, previous studies of infants have focused on the effects of density 


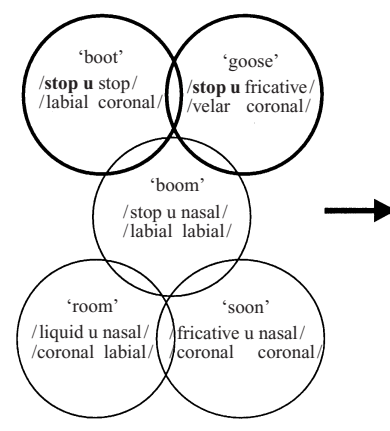

Infant

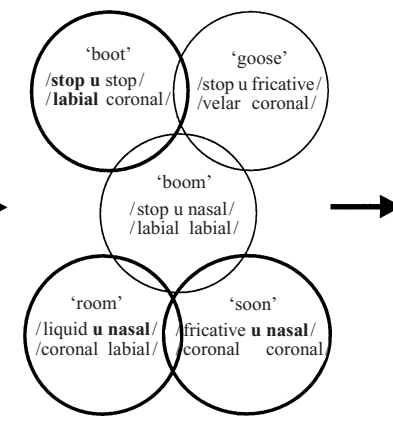

Child

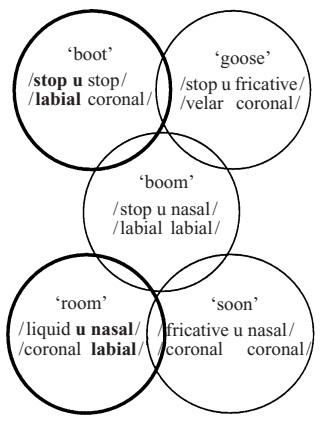

Adult

Fig. 4. Changes predicted by the weak restructuring account for the sparse neighbourhood 'boom' in the infant (left panel), preschool child (middle panel), and adult (right panel) lexicons. Relevant similarity relationships are noted in bold. Salience of similarity relationship are indicated by the weight of the circle (bold vs. plain).

on processing and have generally not examined structure as a function of neighbourhood density. Future investigations involving infants may complement this developmental picture which assumes that neighbourhoods are dynamic, with organizational change throughout lexical development.

\section{Weak restructuring account}

An alternative explanation is that neighbourhoods are static, but the child's focus shifts as different properties of words become salient as the lexicon increases in size (Nittrouer, I996; Smith \& Samuelson, I997). Figure 4 illustrates proposed changes in the lexical representation of 'boom' for infants (left panel), preschool children (middle panel), and adults (right panel) under this weak restructuring account. As shown in Figure 4, the infant, child, and adult neighbourhoods for 'boom', all include 'goose', 'boot', 'soon', and 'room'. What changes across development in this framework is the salience or strength, represented in bold, of particular similarity relationships. We hypothesize that as the lexicon increases in size only particular similarity relationships are relevant for spoken language processing, and this effectively limits the number of neighbours of a word affording efficient access to the word. In the infant neighbourhood for 'boom' (left-most panel), 'boot' and 'goose' appear in bold circles because manner similarity in the onset + nucleus position is thought to be the most salient relationship. During childhood (middle panel), the prominence of phoneme similarity in the onset + nucleus position increases, so that 'goose' is no longer a salient neighbour of 'boom'. This essentially limits the number of words overlapping in the onset + nucleus that compete with 'boom' for access during spoken language processing. At this same time, attention to the 
rhyme position of overlap also increases with manner similarity having primacy over other types of similarity. In this way, 'room' and 'soon' are stronger neighbours of 'boom' than in the infant lexicon. While this change in salience of rhyme overlap may increase the number of words that compete with 'boom', it may also provide a fuller specification of words. Finally, in adulthood (right-most panel), phoneme similarity becomes the most salient similarity relationship in the rhyme position. As in childhood, this change in type of similarity decreases the number of words that compete with 'boom' during spoken language processing.

We have traced the developmental changes that are assumed to occur in a sparse neighbourhood under the weak restructuring account. Presumably, dense neighbourhoods would follow a parallel course of development, but at an accelerated rate due to the greater confusability of words in dense neighbourhoods relative to sparse neighbourhoods.

It appears that both the strong and weak restructuring accounts can adequately account for the available data; however, it may be possible to differentiate the two by appealing to other types of evidence. One of the reported strengths of the weak restructuring account is that it can account for variability across tasks (Smith \& Samuelson, I997). That is, not only does salience shift as a result of increasing the size of the lexicon, but also as a result of task differences. Language learning and task constraints are thus both motivators of change. Given this, the weak restructuring account predicts that if an appropriately sensitive task is used, then evidence of the existence of similarity dimensions that are not developmentally salient may be found. For example, it may be possible to identify a task where infants would demonstrate sensitivity to similarity in the rhyme position of overlap, even though this is not a salient dimension during infancy. Likewise, under certain task circumstances, adults may demonstrate broader neighbourhoods encompassing words that share a coarser coding of similarity than the phoneme. Moreover, the weak restructuring account may be consistent with observed differences in the influence of position of overlap on children's performance on spoken language processing tasks as compared to classification tasks. In particular, perception and production evidence seems to support the salience of the onset in early development, whereas similarity judgments seem to support the salience of the rhyme (Stoel-Gammon \& Dunn, I985; Treiman \& Zukowski, I99 I Jusczyk et al., I999; Swingley et $a l$., I 999). It may be that task constraints support differential reliance on one position of overlap over the other. By comparison, the strong restructuring account would appear to be incompatible with this type of variability because words move out of the neighbourhood when a similarity relationship is refined, or are excluded from the neighbourhood when a similarity relationship is not recognized. To date, there have been no studies that have explored variability as a potential means of disambiguating between these 
competing hypotheses about lexical organization and change. This serves as an important direction for future research.

\section{A final caveat}

While the evidence presented suggests that neighbourhood density influences lexical restructuring, it is important to reiterate that density is correlated with phonotactic probability. A two-representation model may be helpful in disambiguating the effects of density versus phonotactic probability on lexical restructuring. Specifically, it may be useful to assume that speakers have access to lexical representations, as well as phonological representations, namely individual sounds or sound combinations. It has been proposed that neighbourhood density affects lexical processing; whereas, phonotactic probability is thought to affect phonological processing (Vitevitch \& Luce, I 999). In any given context, one type of representation is assumed to be more influential. This proposal has been validated in experiments examining the effect of lexicality on word recognition. That is, word recognition tasks involving nonwords, which have no lexical representation, show facilitory effects of phonotactic probability presumably due to the heavy influence of phonological processing (Vitevitch \& Luce, 1999). In contrast, word recognition tasks involving known words show an inhibitory effect of density seemingly due to competition among lexical representations (Vitevitch \& Luce, I 999).

The current study utilized real words that were familiar to young children and a pre-exposure phase where the words were presented in a story context. For this reason, it seems likely that the current method tapped lexical representations, not phonological representations. Thus, we assert that restructuring of lexical representations may be influenced by density rather than phonotactic probability. A related issue is the influence of other phonological variables on lexical representations. In particular, current models of similarity neighbourhoods assume that membership is blind to syllable structure with positions of overlap crossing levels of syllable structure. For example, an onset + nucleus neighbour shares a constituent of the syllable, the onset, and a constituent of the rhyme, the nucleus. It may be that syllable structure may not play a role in the organization of lexical representations, but may influence the organization of phonological representations. Alternatively, it is possible that syllable structure influences organization of both lexical and phonological representations and that current models of similarity neighbourhoods may need to be revised. It will be important for future work to continue to address these issues by attempting to differentiate lexical from phonological representations. In this regard, it may be helpful to compare tasks using real word stimuli to those using nonword stimuli. 
CONCLUSION

The mental representation of words appears to change with development suggesting that lexical development is characterized by both the acquisition of new words as well as the restructuring of known words. This parallels development in other areas where acquisition of new knowledge appears to promote changes in the mental representation of existing knowledge. Importantly, changes in structure appeared to occur gradually and were influenced by the similarity relationships among items (i.e. density) as well as processing characteristics (i.e. sequential processing). These findings may inform the study of category development more generally by providing evidence of the types of factors that may influence category restructuring.

\section{REFERENCES}

ASHA Committee on Audiologic Evaluation. (1985). Guidelines for identification audiometry. ASHA 27, 49-52.

Brown, R. (1973). A first language: the early stages. Cambridge, MA: Harvard University Press.

Carey, S. (1985). Conceptual change in childhood. Cambridge, MA: MIT Press.

Charles-Luce, J. \& Luce, P. A. (I990). Similarity neighbourhoods of words in young children's lexicons. Fournal of Child Language $\mathbf{1 7}, 205^{-1} 5$.

Charles-Luce, J. \& Luce, P. A. (I 995). An examination of similarity neighbourhoods in young children's receptive vocabularies. Fournal of Child Language 22, 727-35.

Cohen, J. ( I988). Statistical power analysis for the behavioural sciences (2nd edition). Hillsdale, NJ : Erlbaum.

Cole, R. A. ( ( 981). Perception of fluent speech by children and adults. Annals of the New York Academy of Sciences 379, 92-109.

Dinnsen, D. A. \& Elbert, M. (I 984). On the relationship between phonology and learning. In M. Elbert, D. A. Dinnsen \& G. Weismer (eds), Phonological theory and the misarticulating child (ASHA Monographs No. 22 ; pp. 59-68). Rockville, MD: ASHA.

Dollaghan, C. A. (I994). Children's phonological neighbourhoods: half empty or half full? Fournal of Child Language 21, 257-72.

Dunn, L. M. \& Dunn, L. M. (I98I). Peabody Picture Vocabulary Test-Revised. Circle Pines, MN : American Guidance Service.

Elliott, L. L., Hammer, M. A. \& Evan, K. E. (r987). Perception of gated, highly familiar spoken monosyllabic nouns by children, teenagers, and older adults. Perception \& Psychophysics 42, I $50-7$.

Ferguson, C. A. \& Farwell, C. B. (I 975). Words and sounds in early language acquisition: english initial consonants in the first fifty words. Language 5I, 4I 9-39.

Garlock, V. M., Walley, A. C. \& Metsala, J. L. (200I). Age-of-acquisition, word frequency and neighbourhood density effects on spoken word recognition: implications for early reading ability. Fournal of Memory and Language 44, I-25.

Gattuso, B., Smith, L. B. \& Treiman, R. (I99I). Classifying by dimensions and reading: a comparison of auditory and visual modalities. Fournal of Experimental Child Psychology 5I, I 39-69.

Gerken, L. A., Murphy, W. B. \& Aslin, R. N. (r 995). Three- and four-year-olds' perceptual confusions for spoken words. Perception \& Psychophysics $\mathbf{5 7}, 475^{-86}$.

Gierut, J. A. (I996). Categorization and feature specification in phonological acquisition. Fournal of Child Language 23, 397-4 I 5

Goldman, R. \& Fristoe, M. (I 986). Goldman-Fristoe Test of Articulation. Circle Pines, MN : American Guidance Service. 
Gupta, P. \& Dell, G. S. (I 997). The emergence of language from serial order and procedural memory. In B. MacWhinney (ed.), Emergentist Approaches to Language. Hillsdale, NJ: Erlbaum.

Huynh, H. \& Feldt, L. S. (I976). Estimation of the Box correction for degrees of freedom from sample data in randomized block and split-plot designs. Fournal of Educational Statistics $\mathbf{1}, 69-82$.

Jusczyk, P. W., Goodman, M. B. \& Baumann, A. (r999). Nine-month-olds' attention to sound similarities in syllables. Fournal of Memory and Language 40, 62-82.

Kolson, C. J. ( 1962 ). The vocabulary of kindergarten children (Doctoral dissertation, University of Pittsburgh, I960). Dissertation Abstracts 21, 22 I 5.

Logan, J. S. (1 992). A computational analysis of young children's lexicons (Technical Report No. 8). Bloomington, IN : Speech Research Laboratory, Indiana University.

Luce, P. A. (1986). Neighbourhoods of words in the mental lexicon (Research on Speech Perception Technical Report No. 6). Bloomington, IN: Speech Research Laboratory, Indiana University.

Luce, P. A. \& Pisoni, D. B. ( I 998). Recognizing spoken words: the neighbourhood activation model. Ear Eீ Hearing r9, I-36.

Macken, M. A. (г980). The child's lexical representation: the 'puzzle-puddle-pickle' evidence. Fournal of Linguistics $\mathbf{1 6}, \mathbf{I}-\mathbf{I} 7$.

Metsala, J. L. ( I 997). An examination of word frequency and neighbourhood density in the development of spoken-word recognition. Memory Cognition 25, 47-56.

Metsala, J. L. \& Walley, A. C. (r 998). Spoken vocabulary growth and the segmental restructuring of lexical representations: precursors to phonemic awareness and early reading ability. In J. L. Metsala \& L. C. Ehri (eds), Word recognition in beginning literacy. Mahwah, NJ: Erlbaum.

Nittrouer, S. (r996). Discriminability and perceptual weighting of some acoustic cues to speech perception by 3-year-olds. Fournal of Speech and Hearing Research 39, 278-97.

Nusbaum, H. C., Pisoni, D. B. \& Davis, C. K. (r 984). Sizing up the Hoosier mental lexicon (Progress Report No. Iо). Bloomington, IN: Speech Research Laboratory, Indiana University.

Rescorla, L. ( 1989). The language development survey: a screening tool for delayed language in toddlers. Fournal of Speech and Hearing Disorders 54, 587-99.

Reznick, J. S. \& Goldsmith, L. (I989). A multiple form word production checklist for assessing early language. Fournal of Child Language r6, 91-100.

Smit, A. B., Hand, L., Freilinger, J. J., Bernthal, J. E. \& Bird, A. (r990). The Iowa Articulation Norms Project and its Nebraska replication. Fournal of Speech and Hearing Disorders 55, 779-98.

Smith, N. V. (I 973). The acquisition of phonology : a case study. Cambridge, England: C.U.P.

Smith, L. B. \& Samuelson, L. K. (I 997). Perceiving and remembering: category stability, variability and development. In K. Lamberts \& D. R. Shanks (eds), Knowledge, concepts and categories. Studies in cognition. Cambridge, MA: MIT Press.

Stoel-Gammon, C. \& Cooper, J. A. (I984). Patterns of early lexical and phonological development. Fournal of Child Language $\mathbf{I}$, 247-7 I .

Stoel-Gammon, C. \& Dunn, C. (I985). Normal and disordered phonology in children. Austin, TX: Pro-Ed.

Swingley, D., Pinto, J. P. \& Fernald, A. (I999). Continuous processing in word recognition at 24 months. Cognition 7r, 73-108.

Treiman, R. \& Baron, J. (I98I). Segmental analysis ability: development and relation to reading ability. In G. E. MacKinnon \& T. G. Waller (eds), Reading research: advances in theory and practice. New York: Academic Press.

Treiman, R. \& Breaux, M. (I 982). Common phoneme and overall similarity relations among spoken syllables: their use by children and adults. Fournal of Psycholinguistic Research $\mathbf{I}$, 569-98.

Treiman, R. \& Zukowski, A. (r99I). Levels of phonological awareness. In S. A. Brady \& D. P. Shankweiler (eds), Phonological processes in literacy : a tribute to Isabelle Y. Liberman. Hillsdale, NJ : Erlbaum. 
Vitevitch, M. S. \& Luce, P. A. (I 999). Probabilistic phonotactics and neighbourhood activation in spoken word recognition. Fournal of Memory of Language 40, 374-408.

Vitevitch, M. S., Luce, P. A., Pisoni, D. B. \& Auer, E. T. (r999). Phonotactics, neighbourhood activation, and lexical access for spoken words. Brain and Language 68, 306-I I.

Walley, A. C., Smith, L. B. \& Jusczyk, P. W. (r 986). The role of phonemes and syllables in the perceived similarity of speech sounds for children. Memory Cognition 14, 220-9.

Walley, A. C. (I988). Spoken word recognition by young children and adults. Cognitive Development 3, $\mathrm{I} 37-65$.

Ward, T. B. \& Vela, E. (r986). Classifying colour materials: children are less holistic than adults. Fournal of Experimental Child Psychology 42, 273-302.

Weiss, D. J. (r99I). A behavioural assumption for the analysis of missing data: The use of implanted zeroes. Fournal of Social Behavior and Personality 6, 955-64.

\section{APPENDIX}

INSTRUCTIONS TO CHILD

The toy wants to get a lot of chips. If the word is a little like the favourite word, you can put the chip in the toy. The toy's favourite word is standard word. Every time you hear standard word, put the chip in the toy. If you don't hear standard word, put the chip in the garbage can. Remember, every time you hear standard word put a chip in the toy. Listen for standard word. 